\title{
Mining Of Deep Web Interfaces Using Multi Stage Web Crawler
}

\author{
Prof. Parvaneh Basaligheh \\ University of Tech and Management Malaysia \\ pbasaligeh@utmcc.my
}

\begin{tabular}{|c|c|}
\hline Article History & Abstract \\
\hline $\begin{array}{l}\text { Article Submission } \\
12 \text { July } 2020 \\
\text { Revised Submission } \\
13 \text { October } 2020 \\
\text { Article Accepted } \\
19 \text { November } 2020 \\
\text { Article Published } \\
31^{\text {st }} \text { December } 2020\end{array}$ & $\begin{array}{l}\text { As deep web develops at an exceptionally high speed, there has been expanded } \\
\text { interest in procedures that help productively find deep-web interfaces. Nonetheless, } \\
\text { because of the huge volume of web assets and the dynamic idea of deep web, } \\
\text { accomplishing wide inclusion and high proficiency is a difficult issue. In this venture } \\
\text { propose a three-stage framework, for proficient reaping deep web interfaces. In the } \\
\text { main stage, web crawler performs website based looking for focus pages with the } \\
\text { assistance of web indexes, trying not to visit an enormous number of pages. To } \\
\text { accomplish more exact outcomes for an engaged slither, Web Crawler positions } \\
\text { websites to organize profoundly applicable ones for a given subject. In the second } \\
\text { stage the proposed framework opens the web pages inside in application with the } \\
\text { assistance of Jsoup API and preprocess it. At that point it plays out the word include } \\
\text { of inquiry in web pages. In the third stage the proposed framework performs } \\
\text { recurrence investigation dependent on TF and IDF. It additionally utilizes a blend of } \\
\text { TF*IDF for positioning web pages. To kill inclination on visiting some exceptionally } \\
\text { applicable connections in shrouded web registries, In this paper we propose plan a } \\
\text { connection tree information structure to accomplish more extensive inclusion for a } \\
\text { website. Venture trial results on a bunch of delegate areas show the deftness and } \\
\text { exactness of our proposed crawler framework, which proficiently recovers deep-web } \\
\text { interfaces from enormous scope destinations and accomplishes higher reap rates } \\
\text { than different crawlers utilizing gullible Bayes calculation. } \\
\text { Keywords: Deep web, two-stage crawler, feature selection, ranking, adaptive } \\
\text { learning }\end{array}$ \\
\hline
\end{tabular}

\section{Introduction}

The deep (or covered up) web alludes to the substance lie behind accessible web interfaces that can't be filed via looking through motors. In light of extrapolations from an investigation done at University of California, Berkeley, it is assessed that the deep web contains roughly 91,850 terabytes and the surface web is just around 167 terabytes in 2003. Later examinations assessed that 1.9 petabytes were reached and 0.3 petabytes were burned-through worldwide in 2007. An IDC report appraises that the absolute of all computerized information made, reproduced, and devoured will arrive at 6 petabytes in 2014. A critical segment of this gigantic measure of information is assessed to be put away as organized or social information in web data sets - deep web makes up about $96 \%$ of all the substance on the Internet, which is 500-550 times bigger than the surface web. These information contain an immense measure of significant data and substances, for example, Infomine, Clusty, Books In Print might be keen on building a record of the deep web sources in a given space, (for example, book). Since these substances can't get to the restrictive web lists of web indexes (e.g., Google and Baidu), there is a requirement for an effective crawler that can precisely and rapidly investigate the deep web information bases.

It is trying to find the deep web information bases, since they are not enlisted with any web crawlers, are normally scantily dispersed, and keep continually evolving. To address this issue, past work has proposed two sorts of crawlers, conventional crawlers and centered crawlers. Nonexclusive crawlers, get every accessible 
structure and can't zero in on a particular point. Centered crawlers, for example, Form-Focused Crawler (FFC) and Adaptive Crawler for Hidden-web Entries (ACHE) can consequently look through online information bases on a particular subject. FFC is planned with connection, page, and structure classifiers for centered creeping of web shapes, and is reached out by ACHE with extra parts for structure separating and versatile connection student.

The connection classifiers in these crawlers assume a critical part in accomplishing higher slithering productivity than the best-first crawler. Be that as it may, these connection classifiers are utilized to foresee the distance to the page containing accessible structures, which is hard to gauge, particularly for the postponed advantage joins (interfaces in the long run lead to pages with structures). Subsequently, the crawler can be wastefully prompted pages without focused structures. Other than effectiveness, quality and inclusion on pertinent deep web sources are likewise testing. Crawler should create an enormous amount of excellent outcomes from the most pertinent substance sources. For evaluating source quality, SourceRank positions the outcomes from the chose sources by registering the arrangement between them.

\section{Literature Review}

In this paper, author proposed, deep web grows at a very fast pace, there has been increased interest in techniques that help efficiently locate deep-web interfaces. However, due to the large volume of web resources and the dynamic nature of deep web, achieving wide coverage and high efficiency is a challenging issue. Here propose a two-stage framework, namely Smart Crawler, for efficient harvesting deep web interfaces. In the first stage, Smart Crawler performs site-based searching for centre pages with the help of search engines, avoiding visiting a large number of pages. To achieve more accurate results for a focused crawl, Smart Crawler ranks websites to prioritize highly relevant ones for a given topic. In the second stage, Smart Crawler achieves fast insite searching by excavating most relevant links with an adaptive link-ranking.[1]

In this paper, author proposed, How to classify and organize the semantic Web services to help users find the services to meet their needs quickly and accurately is a key issue to be solved in the era of service-oriented software engineering. This paper makes full use the characteristics of solid mathematical foundation and stable classification efficiency of naive bayes classification method. It proposes a semantic Web service classification method based on the theory of naive bayes. It elaborates the concrete process of how to use the three stages of Bayesian classification to classify the semantic Web services in the consideration of service interface and execution capacity.[2]

In this paper, author proposed, automated feature selection is important for text categorization to reduce the feature size and to speed up the learning process of classifiers. In this paper, author present a novel and efficient feature selection framework based on the Information Theory, which aims to rank the features with their discriminative capacity for classification. Author first revisit two information measures: Kullback-Leibler divergence and Jeffreys divergence for binary hypothesis testing, and analyze their asymptotic properties relating to type I and type II errors of a Bayesian classifier. [3]

In this paper, author proposed, the rapid growth of the deep web poses predefine scaling challenges for general purpose crawler and search engines. There are increasing numbers of data sources now become available on the web, but often their contents are only accessible through query interface. Here proposed a framework to deal with this problem, for harvesting deep web interface. Here Parsing process takes place. To achieve more accurate result crawler calculate page rank and Binary vector of pages which is extracted from the crawler to achieve more accurate result for a focused crawler give most relevant links with an ranking. This experimental result on a set of representative domain show the agility and accuracy of this proposed crawler framework which efficiently retrieves web interface from large scale sites.[4]

In this paper, author proposed, web develops at a quick pace, there has been expanded enthusiasm for procedures that assistance effectively find profound web interfaces. Be that as it may, because of the expansive 
volume of web assets and the dynamic way of profound web, accomplishing wide scope and high proficiency is a testing issue. Author propose a two-phase system, to be specific Smart Crawler, for productive gathering profound web interfaces. In the primary stage, Smart Crawler performs site-based hunting down focus pages with the assistance of web crawlers, abstaining from going to a substantial number of pages. [5]

In this paper, author proposed, Classification is a data mining technique used to predict group membership for data instances within a given dataset. It is used for classifying data into different classes by considering some constrains. The problem of data classification has many applications in various fields of data mining. This is because the problem aims at learning the relationship between a set of feature variables and a target variable of interest. Classification is considered as an example of supervised learning as training data associated with class labels is given as input. This paper focuses on study of various classification techniques, their advantages and disadvantages.[6]

In this paper, author proposed, Web mining is an important concept of data mining that works on both structured and unstructured data. Search engine initiates a search by starting a crawler to search the World Wide Web (WWW) for documents. Web crawler works in a ordered way to mine the data from the huge repository. The data on which the crawlers were working was written in HTML tags, that data lags the meaning. It was a technique of text mapping. Semantic web is not a normal text written in HTML tags that are mapped to the search result, these are written in Resource description language. The Meta tags associated with the text are extracted and the meaning of content is find for the updated information and give us the efficient result in no time. [7]

In this paper, author proposed, the web stores huge amount of data on different topics. The users accessing web data vastly in now days. The main goal of this paper is to locating deep web interfaces. To locating deep web interfaces uses techniques and methods. This paper is focus on accessing relevant web data and represents significant algorithm i.e. adaptive learning algorithm, reverse searching and classifier. The locating deep web interfaces system works in two stages. In the first stage apply reverse search engine algorithm and classifies the sites and the second stage ranking mechanism use to rank the relevant sites and display different ranking pages. In this paper, author proposed, selecting the most relevant web databases for answering a given query. The existing database selection methods (both text and relational) assess the source quality based on the querysimilarity-based relevance assessment. When applied to the deep web these methods have two deficiencies. First is that the methods are agnostic to the correctness (trustworthiness) of the sources. Secondly, the query based relevance does not consider the importance of the results. These two considerations are essential for the open collections like the deep web. Since a number of sources provide answers to any query, author conjuncture that the agreements between these answers are likely to be helpful in assessing the importance and the trustworthiness of the sources. [8]

\section{Proposed Methodology}

To productively and viably find deep web information sources, Crawler is planned with a three-stage engineering, website finding and in-webpage investigating, as appeared in above Figure. The principal site finding stage finds the most applicable site for an allowed point, the second in-site investigating stage reveals accessible structures from the site and afterward the third stage apply innocent base order positioned the outcome.

In particular, the site finding stage begins with a seed set of locales in a site information base. Seeds locales are competitor destinations given for Crawler to begin slithering, which starts by following URLs from picked seed locales to investigate different pages and different areas. At the point when the quantity of unvisited URLs in the information base is not exactly a limit during the slithering cycle, Crawler performs "turn around looking" of realized deep web locales for focus pages (exceptionally positioned pages that have numerous connects to different areas) and feeds these pages back to the website information base. Site Frontier gets landing page 
URLs from the site information base, which are positioned by Site Ranker to organize profoundly applicable destinations.

The framework proposes a two-stage framework, in particular Smart Crawler, for productive collecting deep web interfaces. In the main stage, Smart Crawler performs site-based looking for focus pages with the assistance of web indexes, trying not to visit an enormous number of pages. To accomplish more precise outcomes for an engaged slither, Smart Crawler positions websites to organize profoundly important ones for a given subject. In the subsequent stage, Smart Crawler accomplishes quick in-site looking by uncovering most applicable connections with a versatile connection positioning. To kill predisposition on visiting some profoundly significant connections in concealed web indexes, we plan a connection tree information structure to accomplish more extensive inclusion for a website. Our test results on a bunch of delegate spaces show the deftness and exactness of our proposed crawler framework, which productively recovers deep-web interfaces from huge scope destinations and accomplishes higher reap rates than different crawlers. Propose a powerful collecting framework for deep-web interfaces, in particular Smart-Crawler. We have demonstrated that our methodology accomplishes both wide inclusion for deep web interfaces and keeps up exceptionally productive creeping. Shrewd Crawler is an engaged crawler comprising of two phases: effective site finding and adjusted in-site investigating. Shrewd Crawler performs webpage based situating by contrarily looking through the realized deep web locales for focus pages, which can adequately discover numerous information hotspots for meager spaces. By positioning gathered locales and by zeroing in the creeping on a point, Smart Crawler accomplishes more exact outcomes.

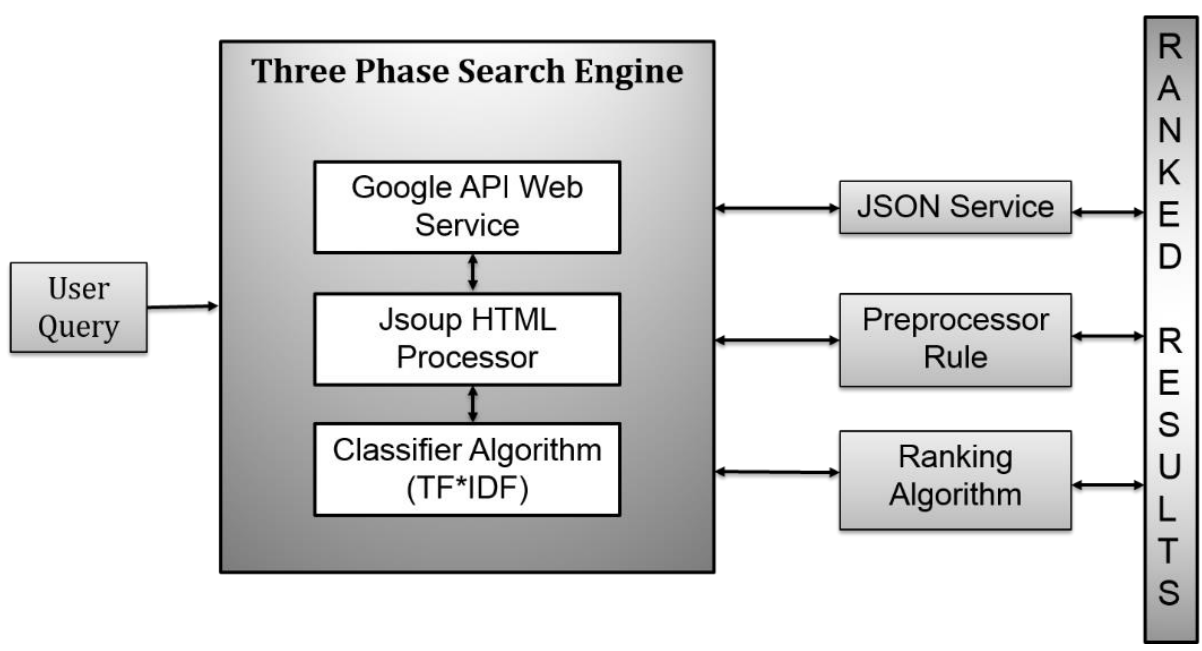

Fig 1. System Architecture

The Site Ranker is improved during crawling by an Adaptive Site Learner, which adaptively learns from features of deep-web sites (web sites containing one or more searchable forms) found. To achieve more accurate results for a focused crawl, Site Classifier categorizes URLs into relevant or irrelevant for a given topic according to the homepage content.

\section{Proposed Algorithm}

- NB algorithm basically works on two different probabilities when it comes to text classification.

- Term Frequency = occur / total

Ex: For a file with 100 works, if a word occurs 10 times

$\mathbf{T F}=\mathbf{1 0} / \mathbf{1 0 0}$

- Inverse Document Frequency = total number of docs / documents in which term occurs

Ex: For 100 files if a word occurs in forty files then,

IDF $=\mathbf{1 0 0} / 40$ 


\section{Conclusion}

In this paper, we propose a compelling reaping framework for deep-web interfaces, in particular Smart-Crawler. We have demonstrated that our methodology accomplishes both wide inclusion for deep web interfaces and keeps up profoundly productive creeping. Smart Crawler is an engaged crawler comprising of two phases: effective site finding and adjusted in-site investigating. Smart Crawler performs website based situating by contrarily looking through the realized deep web destinations for focus pages, which can adequately discover numerous information hotspots for inadequate areas. By positioning gathered destinations and by zeroing in the slithering on a point, Smart Crawler accomplishes more exact outcomes. The in-webpage investigating stage utilizes versatile connection positioning to look inside a website; and we plan a connection tree for killing inclination toward specific catalogues of a website for more extensive inclusion of web indexes. Our exploratory outcomes on a delegate set of spaces show the viability of the proposed two-stage crawler, which accomplishes higher collect rates than different crawlers. In future work, we intend to consolidate pre-question and postinquiry approaches for ordering deep-web structures to additionally improve the precision of the structure classifier.

\section{References}

[1] Feng Zhao, Jingyu Zhou, Chang Nie, Heqing Huang, Hai Jin "Smart Crawler: A Two-stage Crawler for Efficiently Harvesting Deep-Web Interfaces" in IEEE TRANSACTIONS ON SERVICES COMPUTING, VOL. 9, NO. 4, JULY/AUGUST 2016.

[2] Jianxiao Liu, Zonglin Tian, Panbiao Liu, Jiawei Jiang, “An Approach of Semantic Web Service Classification Based on Naive Bayes" in 2016 IEEE International Conference on Services Computing, SEPTEMBER 2016.

[3] Bo Tang, Student Member, IEEE, Steven Kay, Fellow, IEEE, and Haibo He, Senior Member, IEEE "Toward Optimal Feature Selection in Naive Bayes for Text Categorization" in IEEE TRANSACTIONS ON KNOWLEDGE AND DATA ENGINEERING, 9 Feb 2016.

[4] Amruta Pandit, Prof. Manisha Naoghare, "Efficiently Harvesting Deep Web Interface with Reranking and Clustering”, in International Journal of Advanced Research in Computer and Communication Engineering Vol. 5, Issue 1, January 2016.

[5] Anand Kumar, Rahul Kumar, Sachin Nigle, Minal Shahakar, "Review on Extracting the Web Data through Deep Web Interfaces, Mechanism", in International Journal of Innovative Research in Computer and Communication Engineering, Vol. 4, Issue 1, January 2016

[6] Sayali D. Jadhav, H. P. Channe "Comparative Study of K-NN, Naive Bayes and Decision Tree Classification Techniques" in International Journal of Science and Research, Volume 5 Issue 1, January 2016.

[7] Akshaya Kubba, "Web Crawlers for Semantic Web" in International Journal of Advanced Research in Computer Science and Software Engineering, Volume 5, Issue 5, May 2015.

[8] Monika Bhide, M. A. Shaikh, Amruta Patil, Sunita Kerure,"Extracting the Web Data Through Deep Web Interfaces" in INCIEST-2015.

[9] Y. He, D. Xin, V. Ganti, S. Rajaraman, and N. Shah, "Crawling deep web entity pages," in Proc. 6th ACM Int. Conf. Web Search Data Mining, 2013, pp. 355-364.

[10] Raju Balakrishnan, Subbarao Kambhampati, "SourceRank: Relevance and Trust Assessment for Deep Web Sources Based on Inter-Source Agreement" in WWW 2011, March 28-April 1, 2011.

[11] D. Shestakov, "Databases on the web: National web domain survey," in Proc. 15th Symp. Int. Database Eng. Appl., 2011, pp. 179-184. [12] D. Shestakov and T. Salakoski, "Host-ip clustering technique for deep web characterization," in Proc. 12th Int. Asia-Pacific Web Conf., 2010, pp. 378-380.

[12] S. Denis, "On building a search interface discovery system," in Proc. 2nd Int. Conf. Resource Discovery, 2010, pp. 81-93.

[13] D. Shestakov and T. Salakoski, "On estimating the scale of national deep web," in Database and Expert Systems Applications. New York, NY, USA: Springer, 2007, pp. 780-789.

[14] Luciano Barbosa, Juliana Freire "An Adaptive Crawler for Locating Hidden Web Entry Points" in WWW 2007. 
[15] K. C.-C. Chang, B. He, and Z. Zhang, "Toward large scale integration: Building a metaquerier over databases on the web," in Proc. 2nd Biennial Conf. Innovative Data Syst. Res., 2005, pp. 44-55.

[16] M. K. Bergman, "White paper: The deep web: Surfacing hidden value," J. Electron. Publishing, vol. 7, no. 1, pp. 1-17, 2001. 